\title{
Estudos de custo da Doença de Parkinson no Brasil: uma lacuna científica evidente
}

\author{
Parkinson's Disease cost studies in Brazill: an evident scientific gap \\ Estudios de costos de la enfermedad de Parkinson en Brasil: una brecha científica evidente
}

\author{
Sávio Luís Oliveira da Silva \\ ORCID: https://orcid.org/0000-0002-1262-8239 \\ Universidade Federal Fluminense, Brasil \\ Universidade de Vassouras, Brasil \\ E-mail: savio.academico@gmail.com \\ Osvaldo Luiz Gonçalves Quelhas \\ ORCID: https://orcid.org/0000-0001-6816-1677 \\ Universidade Federal Fluminense, Brasil \\ E-mail: osvaldoquelhas@id.uff.br \\ Júlio Vieira Neto \\ ORCID: https://orcid.org/0000-0001-5974-7086 \\ Universidade Federal Fluminense, Brasil \\ E-mail: julion@id.uff.br \\ Marco Antônio Araújo Leite \\ ORCID: https://orcid.org/0000-0003-1447-3696 \\ Universidade Federal Fluminense, Brasil \\ E-mail: maaraujoleite@id.uff.br
}

\begin{abstract}
Resumo
O impacto financeiro da Doença de Parkinson na sociedade tem sido discutido amplamente nas últimas décadas e espera-se que aumente no futuro, juntamente com o envelhecimento da população. Contudo, a escassez de estudos de custo da doença no Brasil dificulta a realização de previsões e cálculos de estimativas futuras, prejudicando a avaliação do impacto econômico na vida do indivíduo e nos sistemas de saúde, tanto público quanto privado. O objetivo desta pesquisa é verificar se a escassez de estudos dessa natureza no Brasil é uma realidade, através de um levantamento sobre os estudos de custo com o tratamento da Doença de Parkinson realizados nos últimos anos. A revisão de literatura, realizada com base na busca por palavras-chave sobre o tema, identificou 27 artigos que atendiam aos critérios de inclusão e aos filtros de refinamento aplicados sobre o portfólio inicial. Destes somente dois estudos foram realizados no Brasil (um artigo original e uma revisão sistemática), o que comprova a falta de estudos sobre o tema no país.
\end{abstract}

Palavras-chave: Doença de Parkinson; Custo da doença; Avaliação econômica em saúde; Sistemas de saúde.

\begin{abstract}
The financial impact of Parkinson's Disease on society has been widely discussed in recent decades and is expected to increase in the future, along with an aging population. However, the scarcity of studies on the cost of the disease in Brazil makes it difficult to carry out forecasts and calculations of future estimates, impairing the assessment of the economic impact on the individual's life and on health systems, both public and private. The objective of this research is to verify whether the scarcity of studies of this nature in Brazil is a reality, through a research of cost studies with the treatment of Parkinson's Disease carried out in recent years. A systematic literature review, based on a search for keywords on the topic, identified 27 articles that met the inclusion criteria and refinement filters applied to the initial portfolio. Of these, only two studies were carried out in Brazil (an original article and a systematic review), which proves the lack of studies on the subject in the country.
\end{abstract}

Keywords: Parkinson's disease; Cost of illness; Health economic; Health systems.

\section{Resumen}

El impacto financiero de la enfermedad de Parkinson en la sociedad se ha debatido ampliamente en las últimas décadas y se espera que aumente en el futuro, junto con el envejecimiento de la población. Sin embargo, la escasez de estudios sobre el costo de la enfermedad en Brasil dificulta la realización de pronósticos y cálculos de estimaciones futuras, lo que perjudica la evaluación del impacto económico en la vida del individuo y en los sistemas de salud, tanto públicos como privados. El objetivo de esta investigación es verificar si la escasez de estudios de esta naturaleza en Brasil es una realidad, a través de una encuesta de estudios de costos con el tratamiento de la Enfermedad de Parkinson realizada en los últimos años. La revisión de la literatura, basada en una búsqueda de palabras clave sobre el tema, identificó 27 artículos que cumplieron con los criterios de inclusión y filtros de refinamiento aplicados al portafolio inicial. De estos, 
solo dos estudios se realizaron en Brasil (un artículo original y una revisión sistemática), lo que demuestra la falta de estudios sobre el tema en el país.

Palabras clave: Enfermedad de Parkinson; Costo de la enfermedad; Evaluación económica em salud; Sistemas de salud.

\section{Introdução}

Os distúrbios neurológicos estão entre as principais causas de incapacidade na população e representam um grande ônus para a saúde pública, pois são incuráveis em sua maioria e pioram ao longo do tempo (Gaskin et al, 2017). De acordo com a Organização Mundial da Saúde (OMS), há a estimativa de que os distúrbios neurológicos e suas seqüelas afetem cerca de um bilhão de pessoas em todo o mundo (Jaglal et al, 2014).

A doença de Parkinson (DP) é uma doença neurodegenerativa crônica e progressiva, caracterizada por declínio da função motora e não motora, e está frequentemente associada a várias complicações (Liu et al, 2021). Classicamente, dentre os sintomas mais corriqueiros da DP estão o tremor em repouso, rigidez, dificuldade para deambular, déficit no equilíbrio, bradicinesia, declínio intelectual, diminuição na amplitude do movimentos e distúrbios na cognição (Marques et al, 2020).

Considerada como a segunda doença neurodegenerativa mais frequente, após o Alzheimer, afeta todas as populações, independente da etnia, classe social e profissão, havendo certa predominância no sexo masculino (Viana, Pereira \& Vital, 2021).

A cronicidade dessa condição e a falta de tratamento fazem com que sua projeção de carga econômica cresça substancialmente nas próximas décadas, impactando os pacientes e suas famílias, o sistema de saúde e a sociedade como um todo (Prado Jr \& Jamora, 2020).

De acordo com o Neurological Health Charities of Canada (NHCC), o impacto dos distúrbios neurológicos em indivíduos, famílias e sociedades é impressionante, e esses impactos aumentarão, à medida que as condições neurológicas se tornarem as principais causas de morte e incapacidade nos próximos vinte anos (Versnel et al, 2013). Embora os custos econômicos sejam altos, as necessidades médicas e sociais dos pacientes, bem como os custos sociais também são insuficientemente conhecidos (Kruse et al, 2021).

O impacto financeiro da DP na sociedade tem sido discutido amplamente nas últimas décadas e espera-se que aumente no futuro (Gil Prieto et al, 2016). A determinação dos custos de uma doença facilita o entendimento do seu impacto para a sociedade e para os sistemas de saúde, avaliando seu grau de eficiência e entendendo como o mercado tende a se organizar em relação a certos valores (Bovolenta et al, 2017a). Atualmente, não há dados robustos de longo prazo sobre os custos do tratamento de pacientes com doença de Parkinson (Weir et al, 2018).

A escassez de estudos de custo da DP no Brasil dificulta a realização de previsões e cálculos de estimativas futuras, dificultando a avaliação do impacto econômico da DP na vida do indivíduo e nos sistemas de saúde, tanto público quanto privado.

O objetivo desta pesquisa é verificar se a escassez de estudos dessa natureza no Brasil é uma realidade, através de um levantamento sobre os estudos de custo com o tratamento da Doença de Parkinson realizados nos últimos anos.

\section{Metodologia}

A revisão sistemática da literatura foi realizada em outubro de 2021, através dos motores de busca, SCOPUS, PubMed, Medline e Web of Science - com o objetivo de selecionar artigos originais e de revisão sobre o custo da DP. As palavras-chave foram delimitadas segundo três eixos temáticos - Custo da Doença, Doença de Parkinson e Sistemas de Saúde - e organizadas da seguinte forma: ("costs" OR "cost of ilness" OR "cost of disease" OR "health economics" OR "health expenditures" OR "cost analysis") AND ("Parkinson" OR "Parkinson's disease") AND ("health care system" OR "public health care system" OR 
"private health care system"). Esse método identificou 888 documentos nas bases de dados citadas, que foram selecionadas e catalogadas através do software Zotero®, possibilitando a formação de um banco de dados inicial, onde todas as informações pudessem ser visualizadas.

Com os documentos catalogados, realizou-se um filtro inicial com o intuito de remover todos os documentos que não fossem artigos científicos (como anais de congresso, capítulos de livro e protocolos de estudo, por exemplo), excluindo-se ao final deste processo 21 documentos. Em seguida, aplicou-se um segundo filtro, eliminando os artigos duplicados inter ou intra bases. Como resultado deste filtro, foram excluídos 41 artigos duplicados. Foi realizado um terceiro filtro com o objetivo de remover artigos que estavam mais distantes do tema central da pesquisa, realizando uma leitura dos títulos e resumos. Nessa análise foram considerados o contexto da pesquisa e cada eixo temático, e como resultado foram excluídos 766 artigos.

O critério de inclusão contemplou somente artigos que se referiam a custos relacionados a DP e/ou ao uso de medicamentos. Trabalhos que comparassem procedimentos e/ou medicamentos, tratassem de terapias específicas e/ou alternativas, cirurgias, ou ainda relacionados com cuidadores, foram excluídos. Desta forma, aplicou-se o quarto filtro e foram excluídos 29 artigos.

Em consequência dos filtros aplicados, o portfólio de artigos foi reduzido a 31 documentos alinhados com o tema de pesquisa e seus respectivos eixos temáticos. Destes, 4 não estavam escritos em inglês ou português, e consequentemente foram excluídos, consolidando o portfólio em 27 artigos que atendiam aos critérios de pesquisa.

Os artigos selecionados foram lidos completamente por um dos autores desta revisão, e analisados segundo os seguintes critérios: país onde foi realizado o estudo, amostra, design, tipo de custo estudado, perspectiva e valores encontrados. Os resultados foram organizados na Tabela 1.

\section{Conceitos básicos em Avaliação Econômica em Saúde (AES)}

Antes de apresentar os resultados do estudo, torna-se importante abordar alguns conceitos básicos inerentes à uma Avaliação Econômica em Saúde (AES). A função básica de qualquer AES é identificar, medir, avaliar e comparar os custos (Bovolenta et al, 2017b). Nesta análise, é importante identificar o tipo de AES, definir o design do estudo, classificar os custos e definir a perspectiva a ser adotada.

\subsection{Tipos de AES}

- Custo-minimização: dificilmente são utilizados nas avaliações econômicas em saúde, uma vez que exigem que diferentes intervenções produzam iguais consequências, comparando-se apenas os custos (Ministério da Saúde, 2008). A principal vantagem desta abordagem é a simplicidade, uma vez que reduz tempo e recursos em relação a uma avaliação econômica completa (Schlatter, 2016).

- Custo-efetividade: é um processo que visa determinar, de forma sistemática e objetiva, a relação entre os custos e os benefícios decorrentes de intervenções preventivas (Moraz et al, 2015). Na avaliação de custo-efetividade os custos são confrontados com os desfechos clínicos na intenção de entender o impacto de diferentes alternativas, identificando as com melhores efeitos do tratamento, em geral, em troca de um custo menor (Secoli et al, 2010).

- Custo-utilidade: neste tipo de AES, o aspecto mais importante é que as consequências de uma intervenção não são medidas apenas em quantidade de vida (anos de vida), mas também em qualidade de vida, ou seja, considera-se o nível de bemestar do indivíduo (Vanni et al, 2009). Recomenda-se este tipo de avaliação quando as intervenções têm um impacto na sobrevida do paciente ou quando aumentam a qualidade de vida sem alterar as condições de sobrevida, utilizando-se de um desfecho 
genérico que permita comparações entre diferentes condições e intervenções, facilitando a alocação de recursos com base na maximização dos ganhos em saúde (Schlatter, 2016).

- Custo-benefício: as análises de custo-benefício medem tanto os custos como as consequências (ou benefícios) das intervenções, e tradicionalmente ocupam uma posição de destaque nas AES, especialmente entre os economistas e gestores, por ser considerada a mais abrangente e que realmente contempla todos os aspectos da eficiência alocativa, envolvendo questões sanitárias e não sanitárias de determinado programa ou terapia (Ministério da Saúde, 2008).

\subsection{Design do estudo}

O design do estudo epidemiológico define como a pesquisa será realizada em relação ao método adotado (Bovolenta et al, 2017b). Os desenhos de estudo mais discutidos na pesquisa de DP são os seguintes:

- Prevalência e Incidência: os estudos de prevalência investigam todos os custos associados a determinado problema de saúde, verificados num período de tempo específico - normalmente de um ano (Oliveira, Santos \& Silva, 2014). Já os estudos baseados na incidência, de acordo com Oliveira, Santos e Silva (2014), calculam os custos incorridos com doenças, diagnosticadas em determinado ano, ao longo do ciclo da vida.

- Prospectivo e Retrospectivo: existe uma relação temporal, onde em estudos prospectivos os eventos relevantes ainda não aconteceram, ou seja, estudando o paciente ao longo do tempo, formalizando um sistema de coleta de dados focado no objetivo da pesquisa, como questionários desenhados especificamente para pacientes e / ou seus cuidadores, onde tudo é registrado em "tempo real". Em estudos retrospectivos, todos os eventos já haviam ocorrido quando o estudo foi iniciado. Geralmente são empregados em doenças crônicas de longo prazo, como é o caso da DP.

- Econometric method - esta abordagem (econométrica ou incremental), estima a diferença de custos de duas coortes, pareadas por características demográficas e pela presença de condições crônicas, utilizando métodos advindos da Econometria associado ao método de cálculos de custos, podendo ser aplicado o teste de diferença entre os custos médios ou as análises de regressão multinível (Segel, 2006).

\subsection{Classificação dos custos}

- Custos diretos: incluem o valor de todos os recursos e serviços consumidos ao se prover uma determinada intervenção ou em se tratar os efeitos adversos, bem como outras consequências monetárias presentes ou futuras ligadas a ela (Vanni et al, 2009). Segundo Oliveira, Santos e Silva (2014), podem ser classificados em médicos (consultas, internações, medicamentos etc.) e não-médicos (serviços sociais, modificações na residência para acomodar o paciente, terapias auxiliares etc.)

- Custos indiretos - são associados à perda de produtividade econômica devido à morbidade e à mortalidade, e podem ser medidos por meio de aposentadorias e pensões precoces por perda de renda (Pinto \& Ugá, 2010). Incluem também os cuidados informais, definido por Martinez-Marin et al (2015) como ajuda não remunerada fornecida por familiares ou amigos;

- Custos intangíveis - relacionam-se com a dor, exclusão social e sofrimento dos pacientes e suas famílias, usualmente verificadas por meio de medidas de qualidade de vida (Silva, Silva \& Pereira, 2016). No entanto, essa categoria de custos, sobre a qual não há consenso, é frequentemente omitida devido à dificuldade de quantificação (Pinto \& Ugá, 2010).

\section{4- Definição da perspectiva de estudo}

Nas AES, a escolha da perspectiva é uma decisão metodológica importante, pois determina que tipos de custos serão analisados e como valorá-los (Pinto \& Ugá, 2010). Existem potenciais pagadores, como o paciente, o hospital, o sistema de 
saúde, a saúde suplementar ou mesmo toda a sociedade, e ao optar-se por uma destas perspectivas o escopo da estimação de custos restringe-se ao que efetivamente é de responsabilidade financeira do pagador selecionado (Silva, Silva \& Pereira, 2016).

\section{Resultados e Discussão}

Os resultados obtidos por esta pesquisa serão apresentados ao longo desta seção conforme os passos definidos no método científico. O presente estudo teve como objetivo verificar se a escassez de estudos dessa natureza no Brasil é uma realidade, através de um levantamento sobre os estudos de custo com o tratamento da Doença de Parkinson realizados nos últimos anos. Desta forma, após a pesquisa realizada em quatro diferentes bases de dados, efetuou-se uma triagem obedecendo aos critérios de inclusão determinado pelos autores - de acordo com os eixos temáticos norteadores do tema - e definiu-se um portfólio de 27 artigos (ver Tabela 1).

Os artigos selecionados foram desenvolvidos à luz de diferentes metodologias, em diversos países, consequentemente gerando resultados distintos. Com isso, em uma tentativa de padronizar os resultados obtidos referentes ao custo calculado, todos os valores foram convertidos para dólares americanos (US\$) - segundo cotação da moeda em 28 de outubro de 2021.

Praticamente todos os artigos calcularam os custos da doença sem nomear o tipo de avaliação econômica. Observou-se também uma predominância de estudos realizados com a perspectiva social, provavelmente por serem mais abrangentes e por representarem o interesse público.

Por se tratar de uma doença com longa sobrevida, os estudos com design retrospectivos são os mais comuns para a DP, apesar do viés de memória que pode ser gerado em função do período retroativo. Os estudos prospectivos, com acompanhamento do paciente, são mais caros e demorados, e dependem de análise através de modelos econométricos. Mesmo assim, nos artigos revisados, 3 autores (ver Tabela 1) optaram por este design de estudo. Da mesma forma, os estudos de prevalência são os mais adequados para os custos da DP, uma vez que são realizados a partir de um diagnóstico já estabelecido.

A grande maioria dos estudos constatou um aumento do custo da doença de acordo com a idade do paciente e o grau de progressão e severidade da doença. Por exemplo, no estudo de Winter et al (2010), o custo anual da doença para pacientes diagnosticados nos estágios I-II da escala de Hoen e Yahr era de US\$21.138,00, enquanto os pacientes mais severos, diagnosticados nos estágios II-V da mesma escala tiveram gastos anuais na ordem de US $\$ 35.864,00$. Esta constatação já havia sido feita por Yu et al (2004), onde pacientes na faixa de 65 a 79 anos gastavam US\$7.022,00/ano, enquanto os pacientes com mais de 80 anos gastavam US $\$ 8.267,00 /$ ano.

No estudo de Martinez-Martín et al (2015), os autores estimaram a magnitude em que os sintomas determinaram os custos da DP ao longo de um período de 4 anos. Os custos totais médios aumentaram 92,5\% (de US\$2.428,31 no ano 1 para US \$4.675,00 no ano 4). Estes achados corroboram com os estudos de Jhonson et al (2013b) onde os autores avaliaram o quanto o indivíduo economizaria caso a doença não progredisse, ou progredisse em escalas menores. O cenário hipotético em que a progressão da DP foi interrompida resultou em benefícios monetários líquidos de US $\$ 442.429,00 /$ ano por paciente. Isso significa dizer que reduzir as taxas de progressão da doença poderia produzir benefícios econômicos significativos.

No estudo de Yoritaka et al (2015) a gravidade da doença não influenciou os custos médicos diretos. Os pesquisadores avaliaram estes custos em pacientes com DP em um hospital universitário no Japão, calculando os custos médicos médios diretos mensais em US $\$ 485,74$ dólares por pessoa. No entanto, uma análise multivariada revelou que a idade, a presença de sintomas não-motores (como alucinações) e maior duração da doença aumentaram significativamente o custo médico direto. Ainda neste estudo, um maior tempo de doença foi significativamente correlacionado com maiores custos de hospitalização.

Nos estudos de custos totais, houve uma tendência em considerar os custos diretos e indiretos da DP, uma vez que os custos intangíveis ainda carecem de uma metodologia própria para ser quantificada. Observamos na Tabela 1 que 15 dos 24 
artigos optaram pelo custo total da doença. No estudo de Jhonson et al (2013a) entretanto, os custos indiretos foram considerados apenas sob a ótica das seguradoras e do capital humano, enquanto, em outro estudo, o objetivo de Cubo et al (2005) foi avaliar os custos intangíveis somente através de anos de vida perdidos. Outros 5 estudos avaliaram somente os custos diretos da DP.

Os custos diretos representaram a maior parcela dos custos totais, muito provavelmente por englobar um considerável número de variáveis, tais como medicamentos, internações, consultas ambulatoriais, tratamentos auxiliares, atendimento domiciliar, transporte e equipamentos especiais. Von Campenhausen et al (2010) investigaram os custos da DP em 486 pacientes com base em uma pesquisa realizada em seis países (Áustria, República Tcheca, Alemanha, Portugal, Itália e Rússia), com dados econômicos coletados ao longo de um período de 6 meses. Os custos médios totais por paciente variaram entre US\$3055,55 e US\$11.452,50. Tais variações foram devidas a diferenças nas características do sistema de saúde específicas de cada país, condições macroeconômicas, bem como frequências de uso de recursos. Os custos diretos totalizaram cerca de $60 \%$ a $70 \%$ e os indiretos, cerca de $30 \%$ a $40 \%$ dos custos totais.

O custo da DP impacta de forma considerável o Sistema de Saúde. Mudiyanselage et al (2017) estimaram o custo anual da DP a partir da perspectiva domiciliar, social e do sistema de saúde na Austrália. O custo médio anual por pessoa para o sistema de saúde foi de US $\$ 32.556,00$, sendo o maior componente dos custos relacionados à hospitalização (69\% dos custos totais). Em outro estudo, realizado na Espanha por Gil-Prieto et al (2016), as internações provenientes da DP custaram ao Sistema de Saúde algo na ordem de US\$138.697.858,20 por ano, com uma média de US\$5.060,16 por internação.

Dentre os 27 artigos analisados, somente 2 estudos foram realizados no Brasil, sendo que um deles era uma revisão sistemática. Neste trabalho, realizado por Bovolenta et al (2017a), o custo anual por paciente foi de US\$5.853,50, incluindo US $\$ 3.172,00$ em custos diretos e US $\$ 2.681,50$ em custos indiretos. No entanto este estudo considerou somente os agravos provocados pelos sintomas motores da doença.

A discrepância entre os valores dos custos da DP obtidos nos artigos avaliados deve-se aos diferentes métodos, designs, custo estudado e perspectiva de estudo adotada. Estes resultados ilustram a importância em se definir estas variáveis ao realizar estudos de avaliação econômica. 
Tabela 1 - Artigos selecionados para revisão.

\begin{tabular}{|c|c|c|c|c|c|c|c|}
\hline Autores & País / Região & Ano & $\mathrm{n}$ & Design & Tipo de custo & Perspectiva & Valor / ano (US\$) \\
\hline Yu et al & EUA & 2004 & $\begin{array}{l}14857(65- \\
79 \text { anos) / } \\
5592(\geq 80 \\
\text { anos) }\end{array}$ & $\mathrm{RE}$ & $\mathrm{D} / \mathrm{I}$ & SS & $7.022(65-79$ anos $) / 8.267$ ( $\geq 80$ anos $)$ \\
\hline Cubo et al & Espanha & 2005 & 23.147 & $\mathrm{RE}$ & Int. & G & NA \\
\hline Lindgren et al & Europa (5 países) & 2005 & NA & Rev. & $\mathrm{D} / \mathrm{I}$ & $\mathrm{S} / \mathrm{SS}$ & NA \\
\hline Findley & Reino Unido & 2007 & NA & Rev. & $\mathrm{D} / \mathrm{I}$ & NA & NA \\
\hline Winter et al & Alemanha & 2010 & $\begin{array}{l}145 \text { (ano } \\
2000) / 133 \\
\text { (ano 2004) }\end{array}$ & $\mathrm{RE} / \mathrm{PE}$ & $\mathrm{D} / \mathrm{I}$ & S/SS & 21.138 (HY I-II) a 35.864 (HY II-V) \\
\hline Von Campenhausen et al & Europa (6 países) & 2011 & 486 & $\mathrm{RE} / \mathrm{PE}$ & $\mathrm{D} / \mathrm{I}$ & S & 2.968 a 11.124 \\
\hline Zhao et al & Singapura & 2011 & 195 & $\mathrm{PE}$ & $\mathrm{D} / \mathrm{I}$ & S & 10.129 \\
\hline Kowal et al & EUA & 2013 & 630.000 & $\mathrm{PE}$ & $\mathrm{D} / \mathrm{I}$ & S & 22.800 \\
\hline Johnson et al (1) & EUA & 2013 & 1.151 & $\mathrm{RE}$ & $\mathrm{D} / \mathrm{I}$ & IC & 43.506 \\
\hline Richy et al & EUA & 2013 & 15.846 & $\mathrm{RE} / \mathrm{PE}$ & $\mathrm{D} / \mathrm{I}$ & S & 77.499 (compliant) \\
\hline Johnson et al (2) & EUA & 2013 & NA & Rev. & D/I/Int. & $\mathrm{S} / \mathrm{SS}$ & 36.362 \\
\hline Tamás et al & Hungria & 2014 & 110 & $\mathrm{PE}$ & $\mathrm{D} / \mathrm{I}$ & $\mathrm{S} / \mathrm{CH}$ & 6.831 \\
\hline Martinez-Martin et al & Espanha & 2015 & 174 & $\mathrm{PO}$ & $\mathrm{D} / \mathrm{I}$ & $S$ & $13.724,24 /$ ano 4 \\
\hline
\end{tabular}


Research, Society and Development, v. 10, n. 15, e214101522457, 2021

(CC BY 4.0) | ISSN 2525-3409 | DOI: http://dx.doi.org/10.33448/rsd-v10i15.22457

\begin{tabular}{|c|c|c|c|c|c|c|c|}
\hline Rodriguéz-Blázquez et al & NA & 2015 & NA & Rev. & $\mathrm{D} / \mathrm{I}$ & NA & NA \\
\hline Yoritaka et al & Japão & 2016 & 715 & SPO & $\mathrm{D}$ & $\mathrm{S}$ & 5.828 \\
\hline Garcia Ramos et al & Espanha & 2016 & NA & Rev. & D/I/Int. & $\mathrm{S} / \mathrm{SS}$ & NA \\
\hline Gil-Prieto et al & Espanha & 2016 & 438,513 & $\mathrm{RE} / \mathrm{PE}$ & $\mathrm{D}$ & $\mathrm{S} / \mathrm{SS}$ & $\begin{array}{l}\text { 138.697.858,20 (SS) / 5.060,16 (média por } \\
\text { internação) }\end{array}$ \\
\hline Bovolenta et al (1) & Brasil & 2017 & 260 & $\mathrm{RE} / \mathrm{PE}$ & $\mathrm{D} / \mathrm{I}$ & $\mathrm{S}$ & $5.853,50$ \\
\hline Bovolenta et al (2) & NA & 2017 & NA & Rev. & $\mathrm{D} / \mathrm{I} / \mathrm{Int}$ & NA & NA \\
\hline Mudiyanselage et al & Austrália & 2017 & 87 & $\mathrm{PO}$ & $\mathrm{D}$ & $\mathrm{S} / \mathrm{SS}$ & $24.600(\mathrm{SS})$ \\
\hline Yang; Chen & China & 2017 & 116 & $\mathrm{RE} / \mathrm{PE}$ & $\mathrm{D} / \mathrm{I}$ & $\mathrm{S}$ & $3.225,94$ \\
\hline Gaskin et al & Canadá & 2017 & NA & Rev. & $\mathrm{D} / \mathrm{I}$ & $\mathrm{S} / \mathrm{SS}$ & NA \\
\hline Weir et al & Reino Unido & 2018 & 7.060 & $\mathrm{RE} / \mathrm{PE}$ & $\mathrm{D}$ & SS & 3.716 (ano 1) to 6.021 (ano 10) \\
\hline Koay; Rose; Abdelhafiz & NA & 2018 & NA & Rev. & $\mathrm{D}$ & $\mathrm{S} / \mathrm{SS}$ & $\mathrm{NA}$ \\
\hline Prado Jr; Jamora & Filipinas & 2020 & 33 & $\mathrm{RE} / \mathrm{PE}$ & $\mathrm{D} / \mathrm{I}$ & $\mathrm{S} / \mathrm{SS}$ & 6.175 \\
\hline Liu et al & Japão & 2021 & 3950 & $\mathrm{RE}$ & $\mathrm{D}$ & $\mathrm{S} / \mathrm{SS}$ & 29.504 (2014), 31.425 (2015) e 34.140 (2016) \\
\hline Kruse et al & $\begin{array}{l}\text { França, Alemanha, } \\
\text { Holanda, Portugal, } \\
\text { Reino Unido, Suécia }\end{array}$ & 2021 & 228 & RE & $\mathrm{D} / \mathrm{I}$ & $\mathrm{S}$ & $23.255,81$ (período de 3 meses) \\
\hline
\end{tabular}

Legenda: $\mathrm{SPO}=$ semi-prospectivo, $\mathrm{PO}=$ prospectivo, $\mathrm{PE}=$ prevalente, $\mathrm{RE}=$ retrospectivo, $\mathrm{D}=$ custo Direto, $\mathrm{I}=$ custo Indireto, Int. $=$ custo Intangível, $\mathrm{S}=$ sociedade, $\mathrm{CH}=$ capital humano, $\mathrm{SS}=$ sistema de saúde, HY = escala de Hoen \& Yahr, CS = companhias de seguro, G = governo, NA = não de aplica, Rev. = artigo de revisão

Fonte: Autores. 


\section{Conclusão}

É incontestável a importância em se pesquisar o custo da DP e seus impactos no paciente, família, sociedade e sistemas de saúde. Como mostrado na maioria dos estudos avaliados, identificar os fatores de risco modificáveis associados ao início e à progressão da doença pode facilitar o desenvolvimento de estratégias para mitigar seu ônus, que afeta indivíduos, cuidadores e o sistema de saúde.

Os resultados da pesquisa evidenciam grandes custos com a doença, o que realça a importância de se aprofundar as pesquisas sobre o tema. Tais custos sobrecarregam as finanças individuais e o orçamento familiar (sob a ótica da perspectiva do paciente), os sistemas de saúde público e privado (sob a ótica do terceiro pagador), além de significar um grande ônus social, ocasionado pela limitação de produção da mão de obra e pelas aposentadorias ocasionadas pelo curso da doença.

Os artigos selecionados nesta revisão enfatizaram as análises de custos diretos e indiretos da DP. No entanto, a escassez de estudos analisando os custos intangíveis da doença abre uma lacuna considerável nos estudos de AES. A inclusão ou exclusão de uma categoria de custos pode representar uma enorme diferença no resultado da análise. Porém, a valoração dos custos intangíveis ainda é controversa e depende do desenvolvimento de metodologia própria para esse fim. Sugere-se, como trabalhos futuros, o desenvolvimento de pesquisas que incluam os custos intangíveis nos modelos de custeio.

A escassez de estudos sobre custo da DP no Brasil ficou evidente neste trabalho de revisão sistemática. Este fato dificulta a realização de previsões e cálculos de estimativas futuras, prejudicando a avaliação do impacto econômico da DP na vida do indivíduo e nos sistemas de saúde, tanto público quanto privado. A realização de estudos sobre o custo da DP no Brasil, sob as mais diferentes perspectivas, revela-se como um nicho interessante para pesquisas futuras nas mais diferentes áreas.

A presente pesquisa, incluindo a construção do método de revisão, a busca nas bases de dados, a seleção de artigos, a exportação deste para software Zoteroß e a análise estatística (bibliometria) dos estudos, foi realizada em outubro de 2021. Sendo assim, como em todo trabalho de revisão, houve limitação temporal na coleta de dados, e caso haja novos autores ou pesquisas, esses não farão parte do portfólio de artigos selecionados. Além disso, a percepção dos autores que desenvolveram esta pesquisa é limitada, desde a decisão sobre o alinhamento com o tema ou até mesmo quanto à escolha dos critérios de inclusão e exclusão. Por fim, devido à identificação de publicações baseadas em palavras-chave, é possível que não tenham sido encontradas publicações que correspondam ao foco da pesquisa por não conterem as palavras-chave necessárias em seus títulos ou resumos.

Como sugestão para trabalhos futuros, torna-se necessário a replicação da pesquisa em outras bases de busca, além da utilização de diferentes palavras-chave referentes ao tema de pesquisa.

\section{Agradecimentos}

O presente trabalho foi realizado com apoio da Coordenação de Aperfeiçoamento de Pessoal de Nível Superior - Brasil (CAPES) - Código de Financiamento 001

\section{Referências}

Bovolenta, T. M., Silva, S. M. C. A., Saba, R. A., Borges, V., Ferraz, H. B. \& Felicio, A. C. (2017 a). Average annual cost of Parkinson's disease in São Paulo, Brazil, with a focus on disease-related motor symptoms. Clinical Interventions in Aging 12: p.2095-2108

Bovolenta, T. M., Silva, S. M. C. A., Saba, R. A., Borges, V., Ferraz, H. B. \& Felicio, A. C. (2017 b). Systematic Review and Critical Analysis of Cost Studies Associated with Parkinson's Disease. Hindawi Parkinson's Disease, p.1-11

Brasil. (2008). Ministério da Saúde. 2008. Avaliação econômica em saúde: desafios para gestão no Sistema Único de Saúde. Brasília: Ministério da Saúde

Cubo, E., Alvarez, E., Morant, C., Cuesta, J. P., Martín, P. M., Génova, R. \& Freire, J. M. (2005). Burden of Disease Related to Parkinson's Disease in Spain in the Year 2000. Movement Disorders 20(11): p.1481-1487 
Daneault, J. F., Carignan, B., Sadikot, A. F., Panisset, M. \& Duval, C. (2013). Drug-induced dyskinesia in Parkinson's disease: Should success in clinical management be a function of improvement of motor repertoire rather than amplitude of dyskinesia? BMC Medicine 11(73): p.1-18

Findley, L. J. (2007). The economic impact of Parkinson's disease. Parkinsonism and Related Disorders 13: p.8-12

García-Ramos, R., Valdés, E.L., Ballesteros, L., Jesús, S. \& Mir, P. (2016). The social impact of Parkinson's disease in Spain: Report by the Spanish Foundation for the brain. Neurología 31(6): p.401-413

Gaskin, J., Gomes, J., Darshan, S. \& Krewski, D. (2017). Burden of neurological conditions in Canada. NeuroToxicology 61: p.2-10

Gil-Prieto, R., Pascual-Garcia, R., San-Roman-Montero, J., Martínez-Martin, P., Castrodeza-Sanz, J. \& Gil-de-Miguel, A. (2016). Measuring the Burden of Hospitalization in Patients with Parkinson's Disease in Spain. PLOS one 11(3): p.1-11

Jaglal, S. B., Guilcher, S. J. T., Bereket, T., Kwan, M., Munce, S., Conklin, J., Versnel, J., Packer, T., Verrier, M., Marras, C., Pitzul, K. B., \& Riopelle, R. (2014). Development of a Chronic Care Model for Neurological Conditions (CCM-NC). BMC Health Services Research 14: p.1-12

Johnson, S. J., Diener, M. D., Kaltenboeck, A., Birnbaum, H. G., Grubb, E. \& Siderowf, A. D. 2013. An economic model of Parkinson's disease: implications for slowing progression in the United States. Movement Disorders 28(3): p.319-326 (b)

Johnson, S. J., Kaltenboeck, A., Diener, M. D., Birnbaum, H., Grubb, E., Castelli-Haley, J. \& Siderowf, A. D. (2013). Costs of Parkinson's disease in a privately insured population. PharmacoEconomics 31: p.799-806 (a)

Koay, L., Rose, J. \& Abdelhafiz, A. H. (2018). Factors that lead to hospitalisation in patients with Parkinson disease-A systematic review. International Journal of Clinical Practice 72: p.1-5

Kowal, S. L., Dall, T. M., Chakrabarti, R., Storm, M. V. \& Jain, A. (2013). The current and projected economic burden of Parkinson's disease in the United States. Movement Disorders 28(3): p.311-318

Kruse, C. S., Kretschmer, A., Lipinski, M., Verheyen, D., Mengel, M., Balzer-Geldsetzer, S. \& Lorenzl, et al. (2021). "Resource Utilization of Patients with Parkinson's Disease in the Late Stages of the Disease in Germany: Data from the CLaSP Study". PharmacoEconomics 39(5): p.601-15.

Lindgren, P., von Campenhausen, S., Spottke, E., Siebert, U. \& Dodel, R. (2005). Cost of Parkinson's Disease in Europe. European Journal of Neurology 12(Suppl.1): p.68-73

Liu N., Babazono A., Kim S. A. \& Li Y. (2021). Effect of Care Rehabilitation on Medical Expenses, Care Costs, and Total Costs of Elderly Individuals with Parkinson's Disease. Popul Health Manag. 2021 Mar 9. doi: 10.1089/pop.2020.0316.

Marques, N. G. S., Oliveira, M. I. S., Alves, M. N., Leão, S. S., Souza, S. D. B. \& Lopes, G. S. (2020). Doença de Parkinson: os principais danos causados no indivíduo. Research, Society and Development, 9(10): p.1-13

Martínez-Martin, P., Rodriguez-Blazquez, C., Paz, S., Forjaz, M. J., Frades-Payo, B., Cubo, E., Pedro-Cuesta, J. \& Lizán, L. (2015). Parkinson Symptoms and Health Related Quality of Life as Predictors of Costs: A Longitudinal Observational Study with Linear Mixed Model Analysis. PLOS one 10(12): p.1-16

Moraz, G., Garcez, A. S., Assis, E. M., Santos, J. P., Barcellos, N. T. \& Kroeff, L. R. (2015). Estudos de custo-efetividade em saúde no Brasil: uma revisão sistemática. Ciência \& Saúde Coletiva, 20(10): p.3211-3229

Mudiyanselage, S. B., Watts, J. J., Ochom, J. A., Lane, L., Murphy, A. T., Morris, M. E. \& Iansek, R. (2017). Cost of Living with Parkinson's Disease over 12 Months in Australia: A Prospective Cohort Study. Hindawi Parkinson's Disease, p.1-14

Oliveira, M. L., Santos, L. M. P. \& Silva, E. N. (2014). Bases metodológicas para estudos de custos da doença no Brasil. Rev Nutr 27(5): p.585-595

Pinto, M. \& Ugá, M. A. D. (2010). O custo de doenças tabaco-relacionadas para o Sistema Único de Saúde. Cad Saúde Pública 26(6): p.1234-1245

Prado, Mario Jr. \& Jamora, R. D. (2020). Cost of Parkinson's disease among Filipino patients seen at a public tertiary hospital in Metro Manila. Journal of clinical neuroscience: official journal of the Neurosurgical Society of Australasia 74: p.41-46.

Richy, F. F., Pietri, G., Moran, K. A., Senior, E. \& Makaroff, L. E. (2013). Compliance with Pharmacotherapy and Direct Healthcare Costs in Patients with Parkinson's Disease: A Retrospective Claims Database Analysis. Appl Health Econ Health Policy 11: p.395-406

Rodríguez-Blázquez, C., Forjaz, M. J., Lizán, L., Paz, S. \& Martínez-Martín, P. (2015). Estimating the direct and indirect costs associated with Parkinson's disease. Expert Review of Pharmacoeconomics and outcomes research, p.1-23

Schlatter, R. P. (2016). Estudo de custos em doenças crônicas não transmissíveis: manejo da cardiopatia isquêmica e diagnostico precoce de câncer hereditário. Doctoral Thesis. Federal University of Rio Grande do Sul, Brasil

Secoli, S. R., Nita, M. E., Ono-Nita, S. K. \& Nobre M. (2010). Avaliação de tecnologia em saúde: A análise de custo-efetividade. Arq Gastroenterol 47(4):p.329333

Segel J. E. (2006). Cost-of-Illness studies: a primer. [s.1.]: RTI International. RTI-UNC Center of Excellence in Health Promotion Economics

Silva, E. N., Silva, M. T. \& Pereira, M. G. (2016). Identificação, mensuração e valoração de custos em saúde. Epidemiol Serv Saúde 25(2): p.437-439

Tamás, G., Gulácsi, L., Bereczki, D., Baji, P., Takáts, A., Brodzky, V. \& Péntek, M. (2014). Quality of life and costs in Parkinson's disease: a cross sectional study in Hungary. PLOS one 9(9): p.1-7 
Research, Society and Development, v. 10, n. 15, e214101522457, 2021

(CC BY 4.0) | ISSN 2525-3409 | DOI: http://dx.doi.org/10.33448/rsd-v10i15.22457

Vanni, T., Luz, P.M., Ribeiro, R. A., Novaes, H. M. D. \& Polanczyk, C. A. (2009). Avaliação econômica em saúde: aplicações em doenças infecciosas. Cad. Saúde Pública 25(12): p.2543-2552

Viana, A., Pereira, J. S. \& Vital, R. (2021). Estudo da neuropatia em pacientes com Doença de Parkinson: revisão de literatura. Research, Society and Development 10(7):p.1-15

Von Campenhausen, S., Winter, Y., Silva, A.R., Sampaio, C., Ruzicka, E., Barone, P., Poewe, W., Guekht, A., Mateus, C., Pfeiffer, K. P.,Berger, K., Skoupa, J., Botzel, K., Geiger-Gritsch, S., Siebert, U., Balzer-Geldsetzer, M., Oertel, W.H., Dodel, R. \& Reese, J.P. (2011). Costs of illness and care in Parkinson's disease: an evaluation in six countries. European Neuropsychopharmacology 21: p.180-191

Watts, J. J., McGinley, J. L., Huxham, F., Menz, H. B., Iansek, R., Murphy, A. T., Waller, E. R. \& Morris, M. E. (2008). Cost effectiveness of preventing falls and improving mobility in people with Parkinson Disesase: protocol for an economic evaluation alongside a clinical trial. BMC Geriatrics 8(23): p.1-8

Weir, S., Samnaliev, M., Kuo, T. C., Tierney, T. S., Autiero, S. W., Taylor, R. S. \& Schrag, A. (2018). Short- and Long-Term Cost and Utilization of Health Care Resources in Parkinson's disease in the UK. Movement Disorders 33(6):p. 974- 981

Winter, Y., von Campenhausen, S. \& Brozova, H. (2010). Costs of Parkinson's disease in eastern Europe: a Czech cohort study. Parkinsonism and Related Disorders 16(1): p.51-56

Yang, J. X. \& Chen, L. (2017). Economic burden analysis of Parkinson's disease patients in China. Hindawi Parkinson's Disease, p.1-7

Yoritaka, A., Fukae, J., Hatano, T., Oda, E. \& Hattori, N. (2016). The direct cost of Parkinson disease at Juntendo medical university hospital, Japan. Internal Medicine 55 (2): p.113-119

Yu, W., Ravelo, A., Wagner, T. H. \& Barnett, P. G. (2004). The relationships among age, chronic conditions, and healthcare costs. American Journal of Managed Care 10(12): p.909-916

Zhao, Y. J., Tan, L. C. S., Li, S. C., Au, W. L., Seah, S. H., Lau, P. N., Luo, N. \& Wee, H. L. (2011). Economic burden of Parkinson's disease in Singapore. European Journal of Neurology 18: p.519-526 